\title{
Reliability modelling and maintenance policy optimization for a repairable parallel system
}

\author{
Reza Ahmadi, Shaomin Wu, Amirhossein Sobhani
}

\begin{abstract}
This paper proposes an integrated approach for reliability modelling and maintenance scheduling of repairable parallel systems subject to hidden failures. The system consists of heterogeneous redundant subsystems whose failures are revealed only by inspections. Inspections at periodic times reveal the components state and repair actions are decided by the excursion of a basic state process describing the total number of failed components in each subsystem. Using the standard renewal arguments, the paper aims at minimizing the average cost rate by the joint determination of the optimal inspection interval, the partial repair threshold and the preventive replacement threshold. We illustrate the procedure for the case as the components' lifetimes conform to the Weibull distribution. Numerical examples are used to illustrate the proposed model and the response of the optimal solutions to the model's parameters.
\end{abstract}

Index Terms-Maintenance; Partial repair; Hidden failure; Heterogeneity population; Renewal-reward theorem.

\section{INTRODUCTION}

\section{A. Background}

During past decades, failure modelling and maintenance scheduling of safety systems such as fire detectors, and protective device, has attracted great attention. These safety systems are composed of components in parallel and keeping them at a high availability is crucial. This arises from inherent characteristic of such systems whose failures are revealed only by inspections (known as hidden failures). For practitioners, undetected failures in these systems can be of great concern when costs associated with maintenance are significant and undetected downtime can lead to not only a significant loss of revenue but also grave consequences related to health, safety, and environment. Thus, for such systems, an appropriate failure model and maintenance strategy are essential to respectively assess the probability of the system failure and to increase the system availability. A maintenance action incurs cost, which raises an intriguing question: how can the system be inspected and repaired so that the availability of the system

R. Ahmadi is with the School of Mathematics, Iran University of Science and Technology, Tehran, Iran. E-mail: Ahmadi_Reza@iust.ac.ir.

$\mathrm{S}$. Wu is with the Kent University, Canterbury, United Kingdom. Email: s.m.wu@kent.ac.uk.

A. Sobhani is with the Department of Mathematics, Statistics, and Computer Science, Semnan University, Semnan 19111-35131, Iran. E-mail: a_sobhani@semnan.ac.ir

Suggested citation: R. Ahmadi, S. Wu and A. Sobhani, "Reliability Modeling and Maintenance Optimization for a Repairable System With Heterogeneity Population," in IEEE Transactions on Reliability, doi: 10.1109/TR.2021.3068633

(C) 2021 IEEE. Personal use of this material is permitted. Permission from IEEE must be obtained for all other uses, in any current or future media, including reprinting/republishing this material for advertising or promotional purposes, creating new collective works, for resale or redistribution to servers or lists, or reuse of any copyrighted component of this work in other works.
[1] can be ensured and the relevant maintenance costs can be minimized? This paper attempts to answer those questions by considering some characteristics that have not been addressed or studied in the literature.

\section{B. Novelty and contributions}

The main contributions of this paper are twofold. First, to derive the state-driven mean residual lifetime (SDMRL) function under the heterogeneity assumption. The pleasant features of the explored function are that (i) it considers both the age factor and the effect of components state; (ii) it derives the ordinary mean residual lifetime (MRL) function and other specific models.. The second contribution of the paper is on its development of an integrated decision model, which allows considering different types of repair actions and maintenance policies. In literature, there are many maintenance decision models that are well-developed for the homogeneous population. However, little has been studied for the systems composed of components from heterogeneous populations. When the components of a system are from different populations, which are characterized by different failure rates, the major challenge is to explore a methodology capable of handling such scenarios while providing effective decisionmaking.

\section{Overview}

The paper is organized as follows. Section II conducts a literature review. Section III describes the deterioration model and the maintenance decision mechanism. Using the standard renewal theory arguments, Section IV formulates the average cost rate for optimizing the model with respect to maintenance parameters. In the next Section we demonstrate the generality of our model by showing that several existing models are emerged as special cases. The proposed model and the effect of the model's parameters on the optimal solutions are illustrated in Section VI. In the last Section, the main findings of the model are summarized and future research directions are underlined.

\section{RELATED WORK}

In order to elucidate the contribution of the paper and its positioning in contrast to existing models, we provide a brief literature review of research works, mostly centered on reliability modelling and the aspect of inspection and preventive maintenance policies.

During past decades, enormous reliability indicators including MRL functions [2,3] are adopted for assess the probability 
of failures of multi-component systems composed of components from with the same population. Typically, the MRL of a system is defined as the remaining time to the end of the useful life, that is, $T-t \mid T>t$, where $T$ is the failure time of a system and $t$ is the current time. However, this modelling approach can result in biased estimate of system reliability when the MRL function cannot adapt itself to the variation of condition monitoring data. To reduce this accuracy, authors [e.g. see 4-6] attempt to correct this shortcoming by incorporating historical condition monitoring data $X(t)$ into the MRL:

$$
M R L:=\mathbb{E}(T-t \mid T>t, X(t)) .
$$

For example, Huynh et al. [4] present a preventive maintenance model based on a MRL indicator accommodating the effect of deterioration level via incorporating a damage process. Wang and Zhang [6] formulate a recursive prediction model for the residual life of an aircraft engine, given measured oil monitoring information. Although reliability modelling in literature is well-developed for homogeneous multi-component systems, in practice the system consists of components characterized by different failure patterns. This motivated us to devise a state-driven MRL function for an $n$-component parallel system consisting of heterogeneous components:

$$
m(t ; X(t))=\mathbb{E}(T-t \mid X(t)) ; \quad X(t) \neq n .
$$

The above model is further developed by exploring an integrated maintenance policy when considering joint inspection and threshold-type policy for repairable systems. Essentially the model is an extension of previous works [7-13] whose attention is restricted to inspection policies. Among existing models, for example, Jiang and Jardine [8] propose an optimum inspection scheduling model that outperforms classical optimum checking policies [7]. Berrade [10] proposes a model with a two-phase inspection policy and allows the inspection adapt to the actual state of the system. Golmakani and Moakedi [11] proposes an optimal non-periodic inspection policy for a multi-component repairable system. Keleş et al. [12] schedule (periodic) inspections for a three-state Markovian deteriorating system under two types of repair actions (no action and perfect repair). More recently, Seyedhosseini et al. [13] obtain an optimal periodic inspection interval for a twocomponent system subject to hidden and two-stage revealed failures.

As an extension of the models cited above, recent works [14-19] are developed by integrating both the inspection and preventive replacement policy. For instance, Berrade et al. [14] develop hybrid block replacement and age-based inspection policies for a heterogeneous multi-component protection system. Babishin and Taghipour [16] consider the optimal inspection and preventive replacement policy for a multi-component system subject to soft hidden failures and hard failures. Age and the number of minimal repairs are respectively used as decision variables for replacement of hard-type and softtype components. More recently, Babishin and Taghipour [17] explore an approach to the joint determination of optimal inspection and replacement policy of $k$-out-of- $n$ systems. Similar to the earlier work [16] the number of minimal repairs is used as a basis for replacement. Given partial information,
Ahmadi and $\mathrm{Wu}$ [18] present a new approach to jointly determining optimal inspection and preventive replacement policy for parallel systems. Recently, Qiu et al. [19] proposes an optimal maintenance policy for a two-component system with dependent soft and hard failures.

\section{A NOVEL MOdEL}

\section{A. Justification of the idea}

In contrast to the aforementioned models, our approach allows modelling inspection and also exploring a general repair model including partial repair [20-22, 24, 24-32] with some unprecedented characteristics. This is motivated by the extension of earlier works (e.g., see [33]) that include three types of repair actions (no action, partial repair and corrective replacement) and those models whose attentions are restricted to inspection and perfect repair. This deficiency or restriction particularly for heterogeneous population arises from complicated nature of modelling of repairable systems and complexities of the cost model formulation. In this paper we address these problems with two tools, firstly, incorporating a virtual age process into the underlying decision variable, secondly, the probabilistic modelling of the associated transition mechanism induced by a partial repair action.

We will see the present model with the aforementioned characteristics not only accommodates actual situations, but also allows different scenarios to be explored.

Before proceeding to model developments, we optimize a cost model for preventive maintenance in the following setting. We consider the problem of inspecting and maintaining a multi-component parallel system subject to non-self announcing failures. It is assumed that components of the considered system includes two types of components [34], characterized by two different failure rates. The decision process for repair and maintenance of the system is driven by the excursion of the bivariate counting process $\mathbf{X}(t)=\left(X_{1}(t), X_{2}(t)\right)$ that describe the total number of failed components in both categories up to age $t$. The system state $\mathbf{X}(t)$ is determined by inspections at fixed intervals $[35,36] \Pi=\{k \tau: 1,2, \cdots\}$ and corrective and preventive maintenance actions are carried out in response to the observed system state. In preference to earlier works (e.g. see [33]), the approach in this paper is developed by the inclusion of four kinds of preventive repair actions decided by partitioning the state space $\Omega$ into four exclusive subsets no action $\left(\Omega_{0}\right)$, partial repair action $\left(\Omega_{1}\right)$, preventive replacement $\left(\Omega_{2}\right)$, and corrective replacement $\left(\Omega^{*}\right)$. More specifically, preventive maintenance actions are implemented on the basis of the total number of failed components and the maintenance parameters: partial repair threshold (denoted by $\kappa$ ) and preventive replacement threshold $(\ell)$ as follows: on inspection if the revealed state falls in $\Omega_{0}$, that is, the total number of failed components is less than $\kappa$, the system is not repaired and is left to continue (no action); the system undergoes a partial repair if the failure frequency of components is to be somewhere between $\kappa$ and $\ell-1$; the system is preventively replaced with new one if without a failure the total number of failed components reaches $\ell$. The partial repair model adopted here is similar to that used by some authors (e.g., [33]; the 
effect of a partial repair is reflected in the system state through a change of the time origin. In this way, via a virtual age concept the system state is restored as far back as the system state at the start of the last intervention. However, our model uses a general kernel density governing state translations of a virtual age process. One of the main advantages of the developed approach is that it enables decision makers to address the trade-off between repair level controlled by some repair parameters and repair costs. We address the problem with the further tool that is the standard method of seeking the regeneration points of the stochastic process $\mathbf{X}(t)$. The sequence of regeneration points defined by replacement epochs makes an embedded renewal process and this allows using the renewal-reward theorem and formulating the average cost rate. The average cost rate is used as a measure of inspection and preventive maintenance policy for optimizing the model with respect to maintenance parameters $\left(\tau^{*}, \kappa^{*}, \ell^{*}\right)$.

\section{B. Degradation model}

Consider a parallel system consisting of $n$ independent components classified in $k$ categories. Denote $n_{i}(i=1,2, \cdots, k)$ as the number of components in the $i^{\text {th }}$ category and $C_{j}^{i}$ as the $j^{\text {th }}$ component in the $i^{\text {th }}$ category with lifetime $T_{i j}$ and the corresponding lifetime distribution function $F_{i j}(t)$. This approach is common in application when a heterogeneous system is composed of components with different material, deterioration process, and environmental characteristics.

Lemma 1: Let $\mathbf{X}(t)$ be an $n$-variate counting process with elements $X_{i}(t)$, which counts the total number of failed components in $i^{\text {th }}$ category $(i=1,2, \cdots, k)$ up to age $t$. Then, the transition probability of the state process $\mathbf{X}$ from state $\mathbf{i}=\left(i_{1}, i_{2}, \cdots, i_{k}\right)$ at $v$ to the state $\mathbf{j}=\left(j_{1}, j_{2}, \cdots, j_{k}\right)$ at $\tau$ $(0<v<\tau)$ becomes

$$
\begin{aligned}
\pi_{\mathbf{i j}}(v, \tau) & =\mathbb{P}(\mathbf{X}(\tau)=\mathbf{j} \mid X(v)=\mathbf{i}) \\
& =\prod_{u=1}^{k} B\left(j_{u}-i_{u}, n_{u}-i_{u}, 1-\frac{\bar{F}_{u}(\tau)}{\bar{F}_{u}(v)}\right)
\end{aligned}
$$

where $B(x, m, p)$ represents the binomial density function with parameters $m$ and $p$ :

$$
B(x, m, p)=\left(\begin{array}{c}
m \\
x
\end{array}\right) p^{x}(1-p)^{m-x} .
$$

To facilitate the presentation due to the homogeneity of components in each category, the subscripts $j$ is dropped of $F_{i j}$.

Proof. The result follows from the probability principles and the independence assumption of components.

The following lemma presents a state-driven reliability indicator function that plays a key role in the failure prediction of the system and decision making. The devised indicator shares some features of the ordinary mean residual lifetime function, but it differs through incorporating the basic process $\mathbf{X}(t)$, which reflects the true state of components.

Lemma 2: Let $T_{n: n}$ be the system's lifetime and $m(t ; \mathbf{i})$ denote the state-driven mean residual lifetime (in short SDMRL)

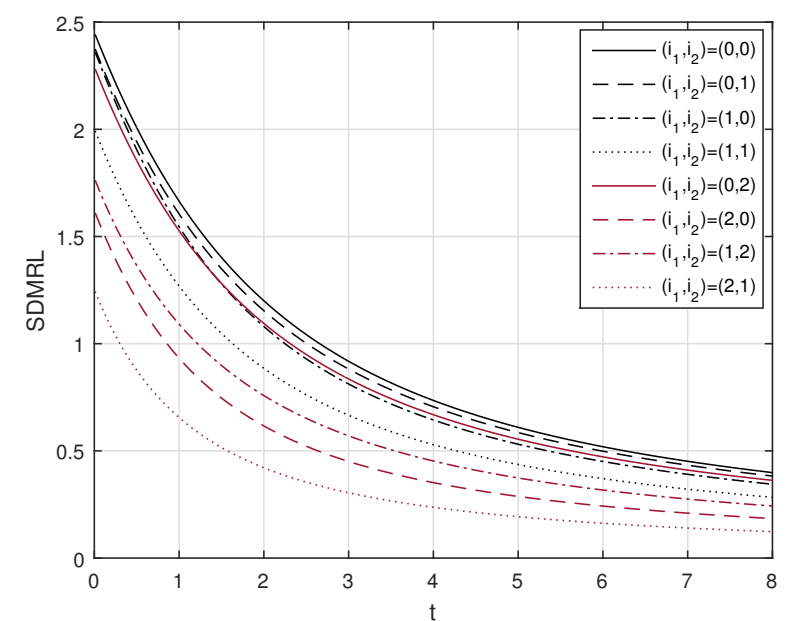

Fig. 1. SDMRL as a function of $t$ given the observed state $\mathbf{X}(t)=\left(i_{1}, i_{2}\right)$.

function of the system at age $t$ given the observed state $\mathbf{X}(t)=\mathbf{i}$, assuming that $i_{0}=\sum_{u=1}^{k} i_{u} \neq n$. In other words,

$$
m(t ; \mathbf{i})=\mathbb{E}\left(T_{n: n}-t \mid \mathbf{X}(t)=\mathbf{i}\right) .
$$

Then,

$$
m(t ; \mathbf{i})=\sum_{v=i_{0}}^{n-1} \sum_{A(v)} \int_{t}^{\infty} \prod_{u=1}^{k} B\left(j_{u}-i_{u} ; n_{u}-i_{u}, 1-\frac{\bar{F}_{u}(w)}{\bar{F}_{u}(t)}\right) d w
$$

where $A(v)$ denotes the set of nonnegative integer solutions to the equation $\sum_{u=1}^{k} j_{u}=v$ :

$$
A(v)=\left\{\mathbf{j}: \sum_{u=1}^{k} j_{u}=v, \quad i_{u} \leq j_{u} \leq n_{u} ; u=1,2, \cdots, k\right\} .
$$

Proof. For proof see Appendix A.

Figure 1 is given to examine the response of the reliability indicator (2) as a function of $t$ for different state values $\mathbf{X}(t)=\left(i_{1}, i_{2}\right)$ when $n_{i}=2(i=1,2)$ and components' lifetimes in category 1 and 2 , respectively, which conform to the Weibull distribution with parameters $\left(\alpha, \beta_{1}\right)=(2,2)$ and $\left(\alpha, \beta_{2}\right)=(2, \sqrt{2})$.

Remark 1: It is sometimes of interest to study of the scaled SDMRL function $g(t ; \mathbf{i})$ for $t \in[0, \infty)$ :

$$
g(t ; \mathbf{i})=\frac{m(t ; \mathbf{i})}{m(0 ; \mathbf{0})} ; \quad g(t ; \mathbf{i}) \in(0,1],
$$

where $m(0 ; \mathbf{0})$ implies the mean time to failure (MTTF) of a heterogenous multi-component parallel system given the starting state $\mathbf{X}(0)=0$. When the system has operated up to time $t$, then $g(t ; \mathbf{i})$ gives the $m(t ; \mathbf{i})$ as a percentage of the initial MMTF.

For illustration purpose, an evolution of the scaled SDMRL function for different state values $\mathbf{X}(t)=\left(i_{1}, i_{2}\right)$ is given (see Figure 2). If, for example, the observed state of failed components in two categories at age $t$ is $\mathbf{X}(t)=\left(i_{1}, i_{2}\right)$ and $g(t ; \mathbf{i})=x$, then the scaled SDMRL is $x \%$ of MTTF at $t=0$ given that $\mathbf{X}(0)=0$.

Before proceeding to the next section, results are developed by considering some specific models emerging as special case. The special cases are described in the following. 


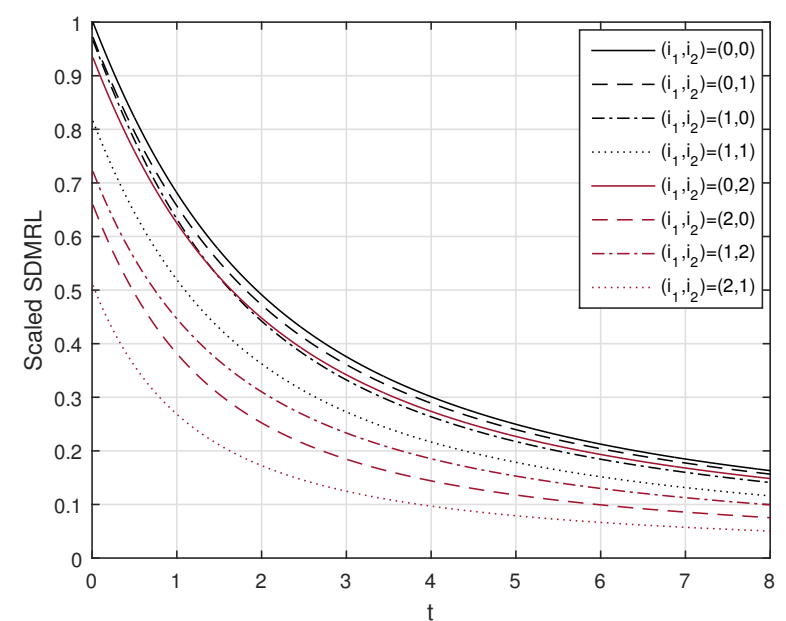

Fig. 2. Scaled SDMRL as a function of $t$ given the observed state $\mathbf{X}(t)=$ $\left(i_{1}, i_{2}\right)$.

Result 3.1: (Homogeneous population) In a particular case, let the homogeneous population be recovered by $k=1$. In this instance, the SDMRL function (2) turns into

$$
m(t ; i)=\sum_{j=i}^{n-1} \int_{t}^{\infty}\left(\begin{array}{c}
n-i \\
j-i
\end{array}\right)\left(1-\frac{F(\omega)}{\bar{F}(t)}\right)^{j-i}\left(\frac{\bar{F}(\omega)}{\bar{F}(t)}\right)^{n-j} d \omega .
$$

Result 3.2: (Exponential homogeneous population) Let $k=$ 1 and the components' lifetimes conform to an exponential distribution with mean value $\lambda$. In this instance, the SDMRL function (2) becomes

$$
m\left(t ; i_{0}\right)=\lambda \sum_{j=i_{0}}^{n-1} \frac{1}{n-j} .
$$

Another important aspect of the explored model (2) is that under the homogeneity assumption, the MRLs function of an $x$-component series system characterizes the SDMRL of a multi-component parallel system.

Proposition 1: Let $k=1$ and $m_{s}(t ; x)$ denote the MRL function of an $x$-component series system. Then, the SDMRL function (4) can be characterized with respect to $m_{s}(t ; x)$ as (6):

$$
m(t ; i)=\sum_{x=1}^{n-i}\left(\begin{array}{c}
n-i \\
x
\end{array}\right)(-1)^{x+1} m_{s}(t ; x) .
$$

Proof. For proof see Appendix B.

It would be of interest to note that in a particular case when components' lifetimes are distributed exponentially with mean value $\lambda$, we have

$$
m(t ; i)=\lambda \sum_{x=1}^{n-i}\left(\begin{array}{c}
n-i \\
x
\end{array}\right)(-1)^{x+1}=\lambda \sum_{x=i}^{n-1} \frac{1}{n-j},
$$

and so,

$$
\sum_{x=1}^{n-i}\left(\begin{array}{c}
n-i \\
x
\end{array}\right)(-1)^{x+1}=\sum_{x=i}^{n-1} \frac{1}{n-j}
$$

Example 1: Let the observed state of an $n$-component homogeneous parallel system be $X(t)=i(i=1,2, \cdots, n-1)$

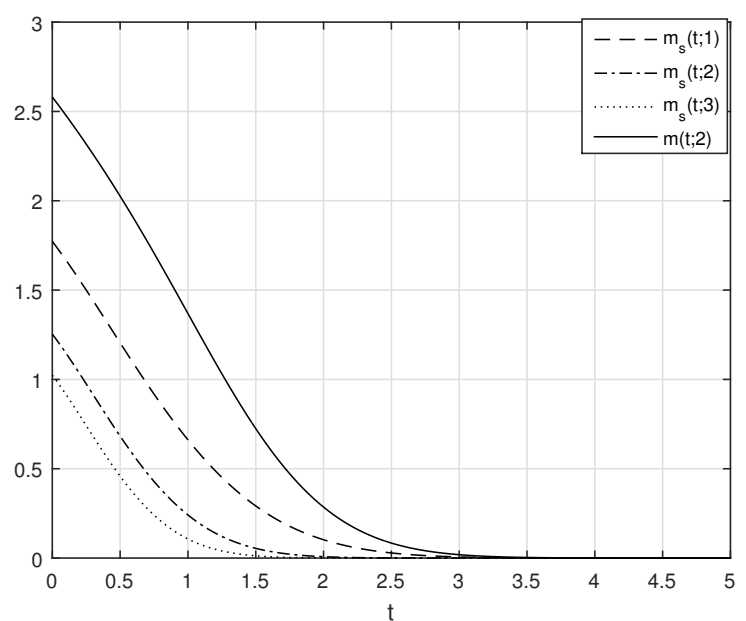

Fig. 3. The decomposed representation of the SDMRL function of an 5component parallel system, $m(t ; 2)$, in terms of the MRL functions of an $x$-component series system $m_{s}(t ; x)(x=1,2,3)$.

and components' lifetimes are distributed with a Weibull distribution with parameters $(\alpha, \beta)=(2, \beta)$. Then,

$$
\begin{aligned}
m(t ; i) & =\beta \sum_{x=1}^{n-i}\left(\begin{array}{c}
n-i \\
x
\end{array}\right)(-1)^{x+1} \sqrt{\frac{\pi}{x}}\left[1-\phi\left(\frac{\sqrt{2 x} t}{\beta}\right)\right] \\
& \times \exp \left(x(t / \beta)^{2}\right) .
\end{aligned}
$$

Figure 3 shows the SDMRL function of an 5-component homogeneous parallel system $m(t ; 2)$ decomposed into the MRL function of an $x$-component series system $m_{s}(t ; x)(x=1,2,3)$ :

$$
m(t ; 2)=3 m_{s}(t ; 1)-3 m_{s}(t ; 2)+m_{s}(t ; 3) .
$$

In contrary fashion, the MRL function of a series system can be decomposed into the SDMRL functions of parallel systems in the following way.

Proposition 2: Under the homogeneity assumption, a decomposed representation of the MRL function of an $i$ component series system in terms of the SDMRL function (4) of an $n$-component parallel system given $X(t)=n-x$ becomes

$m_{s}(t ; i)=\sum_{x=1}^{i}\left(\begin{array}{l}i \\ x\end{array}\right)(-1)^{x+1} m(t ; n-x)$.

Proof. For proof see Appendix C.

Figure 4 indicates the MRL function of an 3-component series system $m_{s}(t ; 3)$, characterized through the SDMRL function of an 5-component parallel system $m(t ; 5-i)(i=1,2,3)$ :

$$
m_{s}(t ; 3)=m(t ; 2)-3 m(t ; 3)+3 m(t ; 4) .
$$

\section{Maintenance model}

In this section, we address the maintenance problem for systems composed of two types of components $(k=2)$. The maintenance decision mechanism described below involves periodic inspections $\Pi=\{\tau, 2 \tau, \cdots\}$. Inspections are perfect and reveal the true state of the bivariate process $\mathbf{X}(t)=$ $\left(X_{1}(t), X_{2}(t)\right) \in \Omega$. Corrective and preventive maintenance 


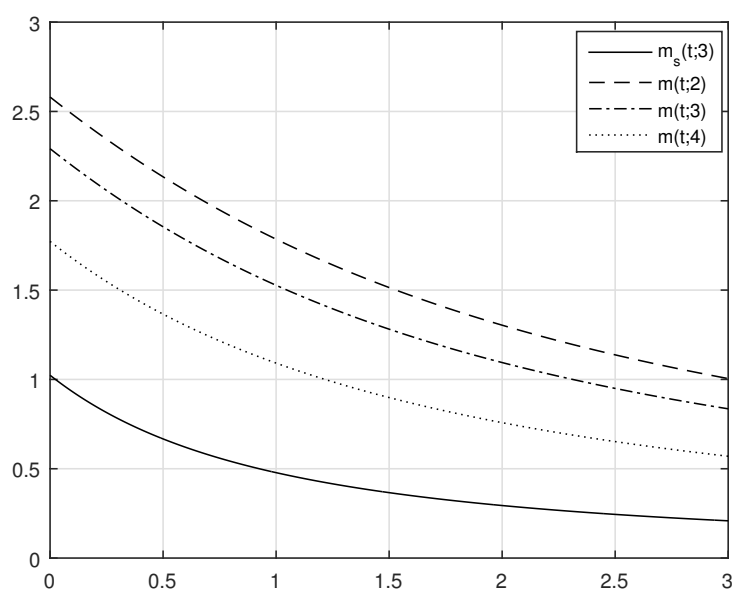

Fig. 4. The decomposed representation of the MRL function of an 3component homogeneous series system in terms of the SDMRLs of an 5component parallel system $m(t ; i)(i=2,3,4)$.

actions are carried out in response to the observed system state. Preventive actions including three kinds of repair actions are decided by partitioning the state space $\Omega$ into non-overlapping sets. Partitioning $\Omega$ is triggered by the maintenance parameters $\kappa$ and $\ell(\kappa<\ell)$, respectively, used as the definition of the partial repair action and the preventive replacement action.

More specifically, assume that the starting state of the system be $\mathbf{X}(0)=\mathbf{0}$ and $\mathbb{A}$ denotes an action matrix of order $\left(n_{1}+1\right) \times\left(n_{2}+1\right)$ with elements $a_{r s}=\langle a, r, s\rangle$ denoting the repair action taken in response to the bivariate state $\left(X_{1}(t), X_{2}(t)\right)=(r, s)$ at age $t$ :

$$
\mathbb{A}=\left[a_{r s}\right]=[\langle a, r, s\rangle]=\left(\begin{array}{cccc}
a_{00} & a_{01} & \ldots & a_{0 n_{2}} \\
a_{10} & a_{11} & \ldots & a_{1 n_{2}} \\
\vdots & \vdots & \ddots & \vdots \\
a_{n_{1} 0} & a_{n_{1} 1} & \ldots & a_{n_{1} n_{2}}
\end{array}\right) .
$$

Further assume that for $0<\kappa<\ell \leq n_{1}+n_{2}-1$

$$
\begin{aligned}
& A_{0}=\{0,1, \cdots, \kappa-1\} \\
& A_{1}=\{\kappa, \kappa+1, \cdots, \ell-1\} \\
& A_{2}=\left\{\ell, \ell+1, \cdots, n_{1}+n_{2}-1\right\}
\end{aligned}
$$

and

$$
\begin{aligned}
& \Omega_{0}=\left\{(r, s) \in \Omega: r+s \in A_{0}\right\} \\
& \Omega_{1}=\left\{(r, s) \in \Omega: r+s \in A_{1}\right\} \\
& \Omega_{2}=\left\{(r, s) \in \Omega: r+s \in A_{2}\right\} \\
& \Omega^{*}=\left\{\left(n_{1}, n_{2}\right)\right\}
\end{aligned}
$$

are the subsets of the state space

$$
\Omega=\left\{(r, s): r=0,1, \cdots, n_{1}, \quad s=0,1, \cdots, n_{2}\right\}
$$

associated with actions $\left\{a_{0}\right\}$ (no action), $\left\{a_{1}\right\}$ (partial repair), $\left\{a_{2}\right\}$ (preventive replacement) and $\left\{a^{*}\right\}$ (corrective replace- ment). Then, $\langle a, r, s\rangle$ with respect to the bivariate state $(r, s)$ can be expressed as

$$
a_{r s}=\langle a, r, s\rangle= \begin{cases}a_{0}, & (r, s) \in \Omega_{0} ; \\ a_{1}, & (r, s) \in \Omega_{1} ; \\ a_{2}, & (r, s) \in \Omega_{2} ; \\ a^{*}, & (r, s) \in \Omega^{*} ;\end{cases}
$$

Therefore the action space including four actions is $\Omega_{a}(4)=$ $\left\{a_{0}, a_{1}, a_{2}, a^{*}\right\}$. Note that in the case that the starting state of the system is $\mathbf{X}(0)=\mathbf{i}=\left(i_{1}, i_{2}\right)$ both the action matrix $A$ and the subsets $\Omega_{i}(i=0,1,2)$ are modified as

$$
\begin{aligned}
& \mathbb{A}(\mathbf{i})=\left[a_{r s}\right] \\
& =[\langle a, r, s\rangle]=\left(\begin{array}{cccc}
a_{i_{1} i_{2}} & a_{i_{1}\left(i_{2}+1\right)} & \ldots & a_{i_{1} n_{2}} \\
a_{\left(i_{1}+1\right) i_{2}} & a_{\left(i_{1}+1\right)\left(i_{2}+1\right)} & \ldots & a_{\left(i_{1}+1\right) n_{2}} \\
\vdots & \vdots & \ddots & \vdots \\
a_{n_{1} i_{2}} & a_{n_{1}\left(i_{2}+1\right)} & \ldots & a_{n_{1} n_{2}}
\end{array}\right) .
\end{aligned}
$$

and

$\Omega_{0}(\mathbf{i})=\left\{(r, s) \in \Omega: r+s \in A_{0}: r \geq i_{1}, s \geq i_{2}\right\}$,

$\Omega_{1}(\mathbf{i})=\left\{(r, s) \in \Omega: r+s \in A_{1}: r \geq i_{1}, s \geq i_{2}\right\}$,

$\Omega_{2}(\mathbf{i})=\left\{(r, s) \in \Omega: r+s \in A_{2}: r \geq i_{1}, s \geq i_{2}\right\}$.

In the present work, the modelling approach is extended by means of a virtual age model. The virtual age process through the change of time origin in bivariate process $\mathbf{X}(t)$ allows the system state to be restored as far back as the system state at the start of the last intervention. More specifically, let $t$ and $\mathbf{X}(t)$ denote the virtual age and the system state just after the last intervention at $(i-1) \tau$. On finding the system in state $\mathbf{X}(t+\tau) \in \Omega_{1}$ at $i \tau$ the decision maker carries out a partial repair which restores the virtual age $(t+\tau) \mapsto V(\tau ; t)$ somewhere between the virtual age after the last intervention, $t$, and the virtual age just before repair, $(t+\tau)$. The effect of the partial repair at $i \tau$ is reflected in the state process through a change of the time origin. In other words, the system found with state $\mathbf{X}(t+\tau)$ is restored to the state $\mathbf{X}(V(\tau ; t))$ where $(V(\tau ; t), \mathbf{X}(V(\tau ; t)))=\rho(t+\tau, X(t+\tau))$. The function $\rho$ describing the repair is a mapping from pre-repair state $X(t+\tau)$ revealed upon inspection at age $(t+\tau)$ to post-repair state induced by a repair action based on exclusive state sets $\Omega_{i}(i=0,1,2)$ and $\Omega^{*}$ :

$\rho(t+\tau, \mathbf{X}(t+\tau))= \begin{cases}(t+\tau, \mathbf{X}(t+\tau)), & \mathbf{X}(t+\tau) \in \Omega_{0} ; \\ (V(\tau ; t), \mathbf{X}(V(\tau ; t))), & \mathbf{X}(t+\tau) \in \Omega_{1} \\ (0,0), & \mathbf{X}(t+\tau) \in \Omega_{2} \\ (0,0), & \mathbf{X}(t+\tau) \in \Omega^{*}\end{cases}$

The approach is extended by the use of a flexible transition density specifying the location of the post-virtual age state $V(\tau ; t) \in(t, t+\tau)$ given the pre-state $(t+\tau)$ :

$$
f_{\tau}(v ; t)=\frac{1}{\tau^{a+b-1}} \times \frac{(v-t)^{a-1}(\tau-v+t)^{b-1}}{\beta(a, b)} ; \quad t<v<t+\tau,
$$

with corresponding expected value

$$
\mathbb{E}(V(\tau ; t))=t+\left(\frac{a}{a+b}\right) \tau,
$$




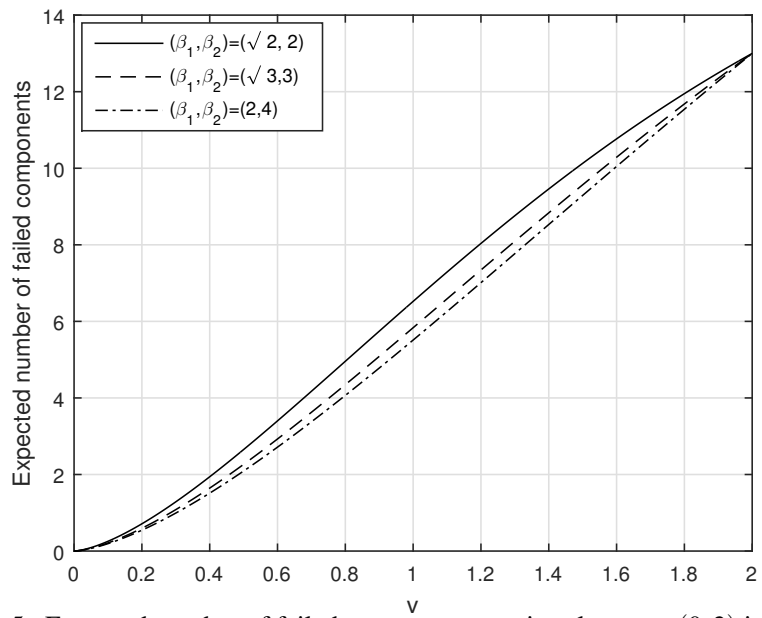

Fig. 5. Expected number of failed components at virtual age $v \in(0,2)$ induced by a partial repair.

where $a>0$ and $b>0$ refer to the parameters of the beta function $B(a, b)$ :

$$
\beta(a, b)=\int_{0}^{1} x^{a-1} b^{b-1} d x .
$$

In the particular case $a=b=1$, we get the uniform distribution with CDF:

$$
F_{\tau}(v ; t)= \begin{cases}0, & v<t \\ \frac{v-t}{\tau}, & v \in(t, t+\tau) \\ 1, & v \geq t+\tau\end{cases}
$$

To facilitate average cost modelling adopted as a measure of policy, Lemma 3 establishes the transition probability of the basic process $\mathbf{X}(t)$, induced by a partial repair action.

Lemma 3: Let $V(\tau ; 0)=v$ denote the updated virtual age, induced by a partial repair at age $\tau$. Then, given the assumption of lemma (1), the transition probability from $\mathbf{X}(\tau)=\mathbf{j}$ to the post-repair state $\mathbf{X}(v)=\mathbf{i}(0<v<\tau)$ becomes

$$
\pi_{\mathbf{j i}}(\tau, v)=\prod_{k=1}^{2} B\left(i_{k} ; j_{k}, \frac{F_{T_{k}}(v)}{F_{T_{k}}(\tau)}\right) .
$$

Proof. It results from lemma 1 and the Bayes' theorem.

Note that under the assumptions of population homogeneity $\left(n_{1}=0\right)$, the transition model turns into

$$
\pi_{j i}(\tau, v)=B\left(i ; j, \frac{F_{T}(v)}{F_{T}(\tau)}\right) .
$$

Assuming that the observed state at intervention time $t=2$ is $\mathbf{X}(t)=(5,8)$, Figure 5 illustrates the response of the expected number of failed components to degradation parameters $\left(\beta_{1}, \beta_{2}\right)$ at virtual age $v \in(0,2)$, induced by a partial repair action. Figure indicates that the expected number of remaining failed components after repair is a decreasing function of degradation parameters $\left(\beta_{1}, \beta_{2}\right)$; as the system becomes more susceptible to failure, few failed components are brought back to the functioning state.

\section{AVERAGe COST RATE}

\section{A. Expected cost per cycle}

A cycle consists of a sequence of inspections and maintenance actions that ultimately ends with (un)planned replacement. Corrective and preventive maintenance actions costs incurred in a cycle are random. Let $C_{\tau}^{\mathbf{i}}$ denote the cost per cycle given starting state $X(0)=\mathbf{i}$, that is, the system starts operating with $\mathbf{n}_{0}=\left(n_{1}-i_{1}, n_{2}-i_{2}\right)$ components of two categories. At inspection time $\tau$, if the bivariate state process $\mathbf{X}(\tau)=(r, s)$ is observed in $\Omega_{0}$ no action is taken, $\langle a, r, s\rangle=a_{0}$, and the system restarts from the current state $\mathbf{X}(\tau) \in \Omega_{0}$. It incurs the planned inspection cost $C_{0}$ and the future costs starting in state $\mathbf{X}(\tau)$. If the system is found in $\Omega_{1}$ and a partial repair is taken, $\langle a, r, s\rangle=a_{1}$, then the system restarts from the post-repair state $\mathbf{X}(V(\tau ; 0))$ with the planned partial repair cost $C(a, b)$ $\left(C(a, b)>C_{0}\right)$ and the future cost starting in state $\mathbf{X}(V(\tau ; 0))$. An additional cost would be incurred if on inspection the revealed state falls in $\Omega_{2}$. In this case the system is returned to the perfect working state, $\langle a, r, s\rangle=a_{2}$, with the replacement cost $C_{r}\left(C_{r}>C(a, b)\right)$ and the future cost starting in state $\mathbf{X}(0)=0$. If the system is found in failed state, $\mathbf{X}(\tau) \in \Omega^{*}$, it undergoes a corrective maintenance, i.e. $\langle a, r, s\rangle=a^{*}$. It incurs an unplanned replacement $\operatorname{cost} C_{f}\left(C_{f}>C_{r}\right)$ and a penalty cost per unit time $C_{p}$ due to an undetected failure within interinspection times. In other words,

$$
\begin{aligned}
& C_{\tau}^{\mathbf{i}}=\left(C_{0}+C_{\tau}^{\mathbf{X}(\tau)}\right) I\left(X(\tau) \in \Omega_{0}(\mathbf{i})\right) \\
& +\left(C(a, b)+C_{\tau}^{\mathbf{X}(V(\tau ; 0))}\right) I\left(\mathbf{X}(\tau) \in \Omega_{1}(\mathbf{i})\right) \\
& +\left(C_{r}+C_{\tau}^{\mathbf{0}}\right) I\left(\mathbf{X}(\tau) \in \Omega_{2}(\mathbf{i})\right)+\left(C_{f}+C_{p}(\tau-T)\right) I\left(\mathbf{X}(\tau) \in \Omega^{*}\right)
\end{aligned}
$$

where $T$ denotes the lifetime of the system, $C(a, b)=p C_{0}+$ $(1-p) C_{p}$ for $p=\frac{a}{a+b} \in(0,1)$ and $C_{\tau}^{\mathbf{0}}$ arises from the preventive replacement which resets all processes to zero. It is noted that the cost function $C(a, b)$ defined as above adapts itself to the repair level, determined by the partial repair parameters $(a, b)$ : higher level of repair induced by smaller (larger) value of the partial repair parameter $a(b)$ incurs more costs.

Taking the expectations of both sides of (10) gives the expected cost per cycle $\mathscr{C}_{\tau}^{\mathbf{i}}(\kappa, \ell)=\mathbb{E}\left(C_{\tau}^{\mathbf{i}}\right)$ :

$$
\begin{aligned}
& \mathscr{C}_{\tau}^{\mathbf{i}}(\kappa, \ell)=\sum_{\mathbf{j} \in \Omega_{0}(\mathbf{i})}\left(C_{0}+\mathscr{C}_{\tau}^{\mathbf{j}}(\kappa, \ell)\right) \pi_{\mathbf{i j}}(0, \tau) \\
& +\left(C_{r}+\mathscr{C}_{\tau}^{\mathbf{0}}(\kappa, \ell)\right) \sum_{\mathbf{j} \in \Omega_{2}(\mathbf{i})} \pi_{\mathbf{i j}}(0, \tau) \\
& +\pi_{\mathbf{i n}_{\mathbf{0}}}(0, \tau)\left(C_{f}+C_{p} \mu\left(\tau ; \mathbf{n}_{\mathbf{0}}\right)\right) \\
& +\int_{0}^{\tau} \sum_{\mathbf{j} \in \Omega_{1}(\mathbf{i})} \sum_{\mathbf{k} \in \Omega(\mathbf{i} \mathbf{j})}\left(C(a, b)+\mathscr{C}_{\tau}^{\mathbf{k}}(\kappa, \ell)\right) \pi_{\mathbf{j k}}(\tau, v) \pi_{\mathbf{i j}}(0, \tau) f_{\tau}(v ; 0) d v
\end{aligned}
$$

where $\mathbf{n}_{0}=\left(n_{1}-i_{1}, n_{2}-i_{2}\right)$,

$\Omega(\mathbf{i}, \mathbf{j})=\left\{(r, s): r+s \leq j_{1}+j_{2}, r=i_{1}, \cdots, j_{1}, s=i_{2}, \cdots, j_{2}\right\}$

and

$$
\mu\left(\tau ; \mathbf{n}_{\mathbf{0}}\right)=\frac{\int_{0}^{\tau} \pi_{\mathbf{i n}_{\mathbf{0}}}(0, u) d u}{\pi_{\mathbf{i n}_{\mathbf{0}}}(0, \tau)},
$$


is the mean past lifetime of an $\left[\left(n_{1}+n_{2}\right)-\left(i_{1}+i_{2}\right)\right]$-component system.

\section{B. Expected cycle length}

Let $L_{\tau}^{\mathbf{i}}$ denote the cycle length starting in $\mathbf{X}(0)=\mathbf{i}$. Using the same argument as above the expected cycle length, $\ell_{\tau}^{\mathbf{i}}(\kappa, \ell)=$ $\mathbb{E}\left(L_{\tau}^{\mathbf{i}}\right)$ is obtained: if at inspection time $\tau$ the bivariate state process $\mathbf{X}(\tau)$ is observed in $\Omega_{0}$, the cycle length consists of an inspection time and an additional cycle length starting from $\mathbf{X}(\tau)$. When finding the system in $\Omega_{1}$ the random time $L_{\tau}^{\mathbf{i}}$ is made up of an inspection time and a cycle length with starting state $\mathbf{X}(V(\tau ; 0))$ is updated after partial repair. In the perfect repair case, the cycle length is made up of a full period $\tau$ and an additional random time $L_{\tau}^{\mathbf{0}}$ starting in state $\mathbf{X}(0)=\mathbf{i}$. On failure at $\tau$ the cycle length is completed. In other words,

$$
\begin{aligned}
L_{\tau}^{\mathbf{i}} & =\left(\tau+L_{\tau}^{\mathbf{X}(\tau)}\right) I\left(X(\tau) \in \Omega_{0}(\mathbf{i})\right) \\
& +\left(\tau+L_{\tau}^{\mathbf{X}(V(\tau ; 0))}\right) I\left(\mathbf{X}(\tau) \in \Omega_{1}(\mathbf{i})\right) \\
& +\left(\tau+L_{\tau}^{\mathbf{0}}\right) I\left(\mathbf{X}(\tau) \in \Omega_{2}(\mathbf{i})\right)+\tau I\left(\mathbf{X}(\tau) \in \Omega^{*}\right)
\end{aligned}
$$

Taking the expectations of both sides of (12) gives the expected cost per cycle $\ell_{\tau}^{\mathbf{i}}(\kappa, \ell)=\mathbb{E}\left(L_{\tau}^{\mathbf{i}}\right)$ :

$$
\begin{aligned}
\ell_{\tau}^{\mathbf{i}}(\kappa, \ell) & =\tau+\sum_{\mathbf{j} \in \Omega_{0}(\mathbf{i})} \ell_{\tau}^{\mathbf{j}}(\kappa, \ell) \pi_{\mathbf{i j}}(0, \tau)+\ell_{\tau}^{\mathbf{0}}(\kappa, \ell) \sum_{\mathbf{j} \in \Omega_{2}(\mathbf{i})} \pi_{\mathbf{i j}}(0, \tau) \\
& +\int_{0}^{\tau} \sum_{\mathbf{j} \in \Omega_{1}(\mathbf{i})} \sum_{\mathbf{k} \in \Omega(\mathbf{i}, \mathbf{j})} \ell_{\tau}^{\mathbf{k}}(\kappa, \ell) \pi_{\mathbf{j k}}(\tau, v) \pi_{\mathbf{i j}}(0, \tau) f_{\tau}(v ; 0) d v .
\end{aligned}
$$

Thus, using the equations (11) and (13), the average cost rate can be given by

$$
\mathbb{C}_{\tau}^{\mathbf{i}}(\kappa, \ell)=\frac{\mathscr{C}_{\tau}^{\mathbf{i}}(\kappa, \ell)}{\ell_{\tau}^{\mathbf{i}}(\kappa, \ell)} .
$$

The optimal period of inspection $\tau^{*}$ and preventive maintenance thresholds $\left(\kappa^{*}, \ell^{*}\right)$ can then be determined as:

$$
\left(\tau^{*}, \kappa^{*}, \ell^{*}\right)=\underset{(\tau, \kappa, \ell) \in(0, \infty) \times \bar{\Omega}}{\operatorname{argmin}} \mathbb{C}_{\tau}^{\mathbf{i}}(\kappa, \ell) .
$$

where $\bar{\Omega}=\Omega \backslash\left\{\left(n_{1}, n_{2}\right)\right\}$.

\section{Obtaining solutions}

The optimization problems above contain equations (11) and (13) so that discretization of the inspection interval with a specific step size $h$ produces corresponding equivalent matrix equations with the general form

$$
\begin{aligned}
(\mathbf{I}-\mathbf{B}) \mathscr{C} & =\mathbf{b}_{c}, \\
(\mathbf{I}-\mathbf{B}) \ell & =\mathbf{b}_{l},
\end{aligned}
$$

where $\mathbf{I}$ is an identity matrix, $\mathbf{B}$ and $\mathbf{b}_{c}, \mathbf{b}_{l}$ are a matrix and two column vectors with known elements and $\mathscr{C}$ and $\ell$ refer to column vectors with elements $\mathscr{C}^{\mathbf{i}}(11)$ and $\ell^{\mathbf{i}}(13)$ respectively. Equations (15a) and (15b) are solved numerically using the function $X=\operatorname{Linsolve}(A, B)$ in matlab solving linear system of equations given in matrix form $A X=B$.

\section{RELATIONSHIP TO OTHER MODELS}

The proposed model covers some maintenance models emerging as special cases. They are recovered by an appropriate choice of decision parameters $(\kappa, \ell)$ or varying other parameters of the model.

\section{A. Variant 1 model: $\Omega_{a}(3)=\left\{a_{0}, a_{2}, a^{*}\right\}$}

The variant 1 repair model corresponds to dropping $\Omega_{1}(\mathbf{i})$ (partial repair action) by setting $\kappa=\ell$. This restricts the action space $\Omega_{a}(4)$ to three kinds of repair actions $\Omega_{a}(3)=$ $\left\{a_{0}, a_{2}, a^{*}\right\}$. The equations (11) and (13) become

$$
\begin{aligned}
& \mathscr{C}_{\tau}^{\mathbf{i}}(\ell)=\sum_{\mathbf{j} \in \Omega_{0}(\mathbf{i})}\left(C_{0}+\mathscr{C}_{\tau}^{\mathbf{j}}(\ell)\right) \pi_{\mathbf{i j}}(0, \tau) \\
& +\left(C_{r}+\mathscr{C}_{\tau}^{\mathbf{0}}(\ell)\right) \sum_{\mathbf{j} \in \Omega_{2}(\mathbf{i})} \pi_{\mathbf{i j}}(0, \tau)+\pi_{\mathbf{i n}_{\mathbf{0}}}(0, \tau)\left(C_{f}+C_{p} \mu\left(\tau ; \mathbf{n}_{\mathbf{0}}\right)\right)
\end{aligned}
$$

and

$$
\ell_{\tau}^{\mathbf{i}}(\ell)=\tau+\sum_{\mathbf{j} \in \Omega_{0}(\mathbf{i})} \ell_{\tau}^{\mathbf{j}}(\ell) \pi_{\mathbf{i j}}(0, \tau)+\ell_{\tau}^{\mathbf{0}}(\ell) \sum_{\mathbf{j} \in \Omega_{2}(\mathbf{i})} \pi_{\mathbf{i j}}(0, \tau) .
$$

\section{B. Variant 2 model: $\Omega_{a}(3)=\left\{a_{0}, a_{1}, a^{*}\right\}$}

The variant 2 repair model with three kinds of actions, i.e. $\Omega_{a}(3)=\left\{a_{0}, a_{1}, a^{*}\right\}$ (excluding preventive replacement action) is recovered by letting $\ell=n$. This turns (11) and (13) into

$$
\begin{aligned}
& \mathscr{C}_{\tau}^{\mathbf{i}}(\kappa, n)=\pi_{\mathbf{i n}_{\mathbf{0}}}(0, \tau)\left(C_{f}+C_{p} \mu\left(\tau ; \mathbf{n}_{\mathbf{0}}\right)\right) \\
& +\sum_{\mathbf{j} \in \Omega_{0}(\mathbf{i})}\left(C_{0}+\mathscr{C}_{\tau}^{\mathbf{j}}(\kappa, n)\right) \pi_{\mathbf{i j}}(0, \tau) \\
& +\int_{0}^{\tau} \sum_{\mathbf{j} \in \Omega_{1}(\mathbf{i})} \sum_{\mathbf{k} \in \Omega(\mathbf{i}, \mathbf{j})}\left(C(a, b)+\mathscr{C}_{\tau}^{\mathbf{k}}(\kappa, n)\right) \pi_{\mathbf{j} \mathbf{k}}(\tau, v) \pi_{\mathbf{i j}}(0, \tau) f_{\tau}(v ; 0) d v
\end{aligned}
$$

and

$$
\begin{aligned}
\ell_{\tau}^{\mathbf{i}}(\kappa, n) & =\tau+\sum_{\mathbf{j} \in \Omega_{0}(\mathbf{i})} \ell_{\tau}^{\mathbf{j}}(\kappa, n) \pi_{\mathbf{i j}}(0, \tau) \\
& +\int_{0}^{\tau} \sum_{\mathbf{j} \in \Omega_{1}(\mathbf{i}) \mathbf{k} \in \Omega(\mathbf{i}, \mathbf{j})} \ell_{\tau}^{\mathbf{k}}(\kappa, n) \pi_{\mathbf{j k}}(\tau, v) \pi_{\mathbf{i j}}(0, \tau) f_{\tau}(v ; 0) d v
\end{aligned}
$$

C. Variant 3 model: $\Omega_{a}(3)=\left\{a_{1}, a_{2}, a^{*}\right\}$

The variant 3 model with three possible actions $\Omega_{a}(3)=$ $\left\{a_{1}, a_{2}, a^{*}\right\}$ (excluding no action) emerges as particular case by letting $\kappa=i_{0}\left(i_{0}=i_{1}+i_{2}\right)$. The assumption reformulates (11) and (13) as

$$
\begin{aligned}
& \mathscr{C}_{\tau}^{\mathbf{i}}\left(i_{0}, \ell\right)=\pi_{\mathbf{i n}_{\mathbf{0}}}(0, \tau)\left(C_{f}+C_{p} \mu\left(\tau ; \mathbf{n}_{\mathbf{0}}\right)\right) \\
& +\left(C_{r}+\mathscr{C}_{\tau}^{\mathbf{0}}(\kappa, \ell)\right) \sum_{\mathbf{j} \in \Omega_{2}(\mathbf{i})} \pi_{\mathbf{i j}}(0, \tau) \\
& +\int_{0}^{\tau} \sum_{\mathbf{j} \in \Omega_{1}(\mathbf{i})} \sum_{\mathbf{k} \in \Omega(\mathbf{i} \mathbf{j})}\left(C(a, b)+\mathscr{C}_{\tau}^{\mathbf{k}}\left(i_{0}, \ell\right)\right) \pi_{\mathbf{j} \mathbf{k}}(\tau, v) \pi_{\mathbf{i j}}(0, \tau) f_{\tau}(v ; 0) d v
\end{aligned}
$$

and

$$
\begin{aligned}
\ell_{\tau}^{\mathbf{i}}\left(i_{0}, \ell\right) & =\tau+\ell_{\tau}^{\mathbf{0}}\left(i_{0}, \ell\right) \sum_{\mathbf{j} \in \Omega_{2}(\mathbf{i})} \mathscr{P}_{\mathbf{i j}}(0, \tau) \\
& +\int_{0}^{\tau} \sum_{\mathbf{j} \in \Omega_{1}(\mathbf{i})} \sum_{\mathbf{k} \in \Omega(\mathbf{i}, \mathbf{j})} \ell_{\tau}^{\mathbf{k}}\left(i_{0}, \ell\right) \mathscr{P}_{\mathbf{j} \mathbf{k}}(\tau, v) \mathbf{P}_{\mathbf{i j}}(0, \tau) f_{\tau}(v ; 0) d v
\end{aligned}
$$


D. Variant 4 model: $\Omega_{a}(2)=\left\{a_{0}, a^{*}\right\}$

The variant 4 model is recovered by merging subsets $\Omega_{i}$ $(i=0,1,2)$ to $\Omega_{0}$ (no action) by assuming $\kappa=\ell=n$. This partitions the action space into two regions associated with no action $\left\{a_{0}\right\}$ and corrective replacement $\left\{a^{*}\right\}$. The equations (11) and (13) become

$$
\begin{aligned}
\mathscr{C}_{\tau}^{\mathbf{i}}(n) & =\sum_{\mathbf{j} \in \Omega_{0}(\mathbf{i})}\left(C_{0}+\mathscr{C}_{\tau}^{\mathbf{j}}(n)\right) \pi_{\mathbf{i j}}(0, \tau) \\
& +\pi_{\mathbf{i n}_{\mathbf{0}}}(0, \tau)\left(C_{f}+C_{p} \mu\left(\tau ; \mathbf{n}_{\mathbf{0}}\right)\right)
\end{aligned}
$$

and

$\ell_{\tau}^{\mathbf{i}}(n)=\tau+\sum_{\mathbf{j} \in \Omega_{0}(\mathbf{i})} \ell_{\tau}^{\mathbf{j}}(n) \pi_{\mathbf{i j}}(0, \tau)$.

\section{E. Variant 5 model: $\Omega_{a}(2)=\left\{a_{1}, a^{*}\right\}$}

The variant 5 repair model with two kinds of actions, i.e. $\Omega_{a}(2)=\left\{a_{1}, a^{*}\right\}$ (excluding no action and preventive replacement action) is recovered by letting $\kappa=i_{0}$ and $\ell=n$. This turns (11) and (13) into

$$
\begin{aligned}
& \mathscr{C}_{\tau}^{\mathbf{i}}\left(i_{0}, n\right)=\pi_{\mathbf{i n}_{\mathbf{0}}}(0, \tau)\left(C_{f}+C_{p} \mu\left(\tau ; \mathbf{n}_{\mathbf{0}}\right)\right) \\
& +\int_{0}^{\tau} \sum_{\mathbf{j} \in \Omega_{1}(\mathbf{i})} \sum_{\mathbf{k} \in \Omega(\mathbf{i}, \mathbf{j})}\left(C(a, b)+\mathscr{C}_{\tau}^{\mathbf{k}}\left(i_{0}, n\right)\right) \pi_{\mathbf{j k}}(\tau, v) \pi_{\mathbf{i j}}(0, \tau) f_{\tau}(v ; 0) d v
\end{aligned}
$$

and

$$
\begin{aligned}
& \ell_{\tau}^{\mathbf{i}}\left(i_{0}, n\right)=\tau \\
& +\int_{0}^{\tau} \sum_{\mathbf{j} \in \Omega_{1}(\mathbf{i})} \sum_{\mathbf{k} \in \Omega(\mathbf{i}, \mathbf{j})} \ell_{\tau}^{\mathbf{k}}\left(i_{0}, n\right) \pi_{\mathbf{j k}}(\tau, v) \pi_{\mathbf{i j}}(0, \tau) f_{\tau}(v ; 0) d v .
\end{aligned}
$$

\section{F. Variant 6 model: $\Omega_{a}(2)=\left\{a_{2}, a^{*}\right\}$}

The variant 6 model corresponds to dropping subsets $\Omega_{0}$ (no action) and $\Omega_{1}$ (partial repair action) from the model by letting $\kappa=\ell=i_{0}\left(i_{0}=i_{1}+i_{2}\right)$. The equations (11) and (13) become

$$
\begin{aligned}
\mathscr{C}_{\tau}^{\mathbf{i}}\left(i_{0}\right) & =\left(C_{r}+\mathscr{C}_{\tau}^{\mathbf{0}}\left(i_{0}\right)\right) \sum_{\mathbf{j} \in \Omega_{2}(\mathbf{i})} \pi_{\mathbf{i j}}(0, \tau) \\
& +\pi_{\mathbf{i n}_{\mathbf{0}}}(0, \tau)\left(C_{f}+C_{p} \mu\left(\tau ; \mathbf{n}_{\mathbf{0}}\right)\right)
\end{aligned}
$$

and

$$
\ell_{\tau}^{\mathbf{i}}\left(i_{0}\right)=\tau+\ell_{\tau}^{\mathbf{0}}\left(i_{0}\right) \sum_{\mathbf{j} \in \Omega_{2}(\mathbf{i})} \pi_{\mathbf{i j}}(0, \tau)
$$

Given the starting state $\mathbf{i}=\mathbf{0}$, the average cost rate becomes

$$
\mathbb{C}_{\tau}^{\mathbf{0}}\left(i_{0}\right)=\frac{C_{r}+F_{T_{s}}(\tau)\left(C_{f}+C_{p} \mu(\tau ; n)\right)}{\tau} .
$$

This is similar to the periodic replacement policy implemented whenever the system reaches age $\tau$ (regeneration instant). The costs in the cycle $(0, \tau)$ are made up from the planned replacement cost $C_{r}$ and the possible additional cost of replacement on failure and a penalty cost incurred due to undetected failure.

\section{NUMERICAL EXAMPLE}

Using the solution procedure IV-C with the step size $h=$ 0.01 , we obtain an optimal solution to maintenance parameters $\left(\tau^{*}, \kappa^{*}, \ell^{*}\right)$. Numerical results are developed by investigating the effect of model's parameters on the optimal solutions.

Let the failure mechanism in both categories be expressed by Weibull distributions. The Weibull distribution function associated with category $i$ is

$$
F_{T_{i}}(t)=1-\exp \left(-\left(t / \beta_{i}\right)^{\alpha_{i}}\right) .
$$

The choice for the degradation parameters is $\left(\alpha_{1}, \beta_{1}\right)=$ $(1.5, \sqrt{2})$ and $\left(\alpha_{2}, \beta_{2}\right)=(1.5,2)$. The transition mechanism of the virtual age process is expressed by the kernel function (8):

$$
f_{\tau}(v ; 0)=\frac{1}{\tau^{a+b-1}} \times \frac{v^{a-1}(\tau-v)^{b-1}}{\beta(a, b)} ; \quad 0<v<\tau,
$$

with $(a, b)=(1,0.5)$. For numerical illustration of the model, we set $C_{0}=0.5, C_{r}=5, C_{f}=8$ and $C_{p}=5$. This characterizes both the expected post-repair state and the partial repair cost as $E(V(\tau ; t))=t+\frac{2}{3} \tau$ and $C(a, b)=2$.

The results summarized in Table I indicate that to reveal the true state of components inspections should be scheduled according to a periodic policy $\Pi^{*}=\left\{k \tau^{*}: k=1,2, \cdots\right\}$ with $\tau^{*}=0.62$ : on inspection if the system is found in state $\Omega_{0}=\{(0,0),(0,1),(1,0),(1,1),(1,2),(2,1)\}$ (at most three of six components experiences failure) the decision maker does not need to take action and leave the system to continue; otherwise the system undergoes a partial repair, or preventive replacement if the system state falls in $\Omega_{1}=\{(2,2)\}$, (two of three components in each category experiences failure) or $\Omega_{2}=\{(2,3),(3,2)\}$. This maintenance policy characterized by optimal solutions $\left(\tau^{*}, \kappa^{*}, \ell^{*}\right)=(0.62,4,5)$ incurs the minimum maintenance cost $\mathbb{C}_{\tau^{*}}^{\mathbf{0}}\left(\kappa^{*}, \ell^{*}\right)=1.47$.

As seen in Table I and Table II, the numerical results are developed by examining the effect of optimal maintenance parameters to the partial repair parameter $a$ and the redundancy level $\left(n_{1}, n_{2}\right)$. The different values for $a \in\{0.5,1,2,4\}$ reflect the decision maker's attitude towards repair. The higher value of $a$ corresponds to almost a minimally repaired system (a risky position) and the higher level of repair corresponds to a certain partial repair bringing the system state back to the condition just after the last intervention. Table I indicates that the optimal preventive replacement threshold $\ell^{*}$ remains constant in all four case, but changes in $a$ induces changes in the optimal inspection policy $\tau^{*}$ and the resulting expected cost per unit time. The model adapts itself to the decision maker's attitudes to repair (the value of $a=4$ ) by moving down the optimal partial repair threshold $\kappa^{*}: 4 \mapsto 3$ : as $a$ increases partial repairs will be considered more often to maintain a minimum level of performance. This results in an increase in the expected cost rate. Also, for illustration purpose an evolution of $\mathbb{C}_{\tau^{*}}^{\mathbf{0}}$ as the function the inspection interval $\tau$ for different redundancy levels $n \in\{6,8\}$ is given by Figure 6 and Figure 7.

Table II indicates that for higher level of redundancy, $n: 6 \mapsto 8$, the optimal parameters respond to the (partial) 
TABLE I

OPTIMAL PARAMETERS FOR DIFFERENT REPAIR LEVELS AND $n_{1}=n_{2}=3$.

\begin{tabular}{llccc}
\hline & \multicolumn{4}{l}{ Optimal maintenance parameters } \\
\cline { 2 - 5 }$a$ & $\kappa^{*}$ & $\ell^{*}$ & $\tau^{*}$ & $\mathbb{C}_{\tau^{*}}^{\mathbf{0}}$ \\
\hline 0.5 & 4 & 5 & 0.63 & 1.38 \\
1.0 & 4 & 5 & 0.62 & 1.47 \\
2.0 & 4 & 5 & 0.62 & 1.55 \\
4.0 & 3 & 5 & 0.60 & 1.61 \\
\hline
\end{tabular}

TABLE II

OPTIMAL PARAMETERS FOR DIFFERENT REPAIR LEVELS AND $n_{1}=n_{2}=4$.

\begin{tabular}{llccc}
\hline & \multicolumn{4}{l}{ Optimal maintenance parameters } \\
\cline { 2 - 5 }$a$ & $\kappa^{*}$ & $\ell^{*}$ & $\tau^{*}$ & $\mathbb{C}_{\tau^{*}}^{\mathbf{0}}$ \\
\hline 0.5 & 5 & 7 & 0.72 & 1.0716 \\
1.0 & 5 & 7 & 0.70 & 1.1619 \\
2.0 & 5 & 7 & 0.67 & 1.2323 \\
4.0 & 5 & 7 & 0.64 & 1.3067 \\
\hline
\end{tabular}

repair parameter $a \in\{0.5,1,2,4\}$ almost in the same way. However, increasing the level of redundancy makes inspections less frequent and gives higher values for the optimal repair thresholds $\kappa^{*}$ and $\ell^{*}$ implying a reduction in the amount of maintenance and the resulting optimal expected cost.

The results in Table III also show the effect of the penalty $\operatorname{cost} C_{p}$ on the optimal maintenance parameters and the optimal expected cost. The results demonstrate that the threshold parameters are not sensitive to $C_{p}$, but as the penalty cost increases, the optimal inspection interval decreases marginally to restrain penalty costs. As shown, in all cases changes in $C_{p}$ induces a slight increase in the optimal expected cost.

Also, the optimal solutions are examined under the general repair model and particular repair models (see Table IV). The results reveal that given repair parameter $(a, b)=(1,0.5)$ only the variant 2 characterized by three possible repair actions $\Omega_{a}=\left\{a_{0}, a_{1}, a^{*}\right\}$ is economically preferable to the general repair model, however, in contrast to the general repair model, inspections are scheduled less frequently. Furthermore, in the absence of the preventive replacement action $\left\{a_{2}\right\}$, the

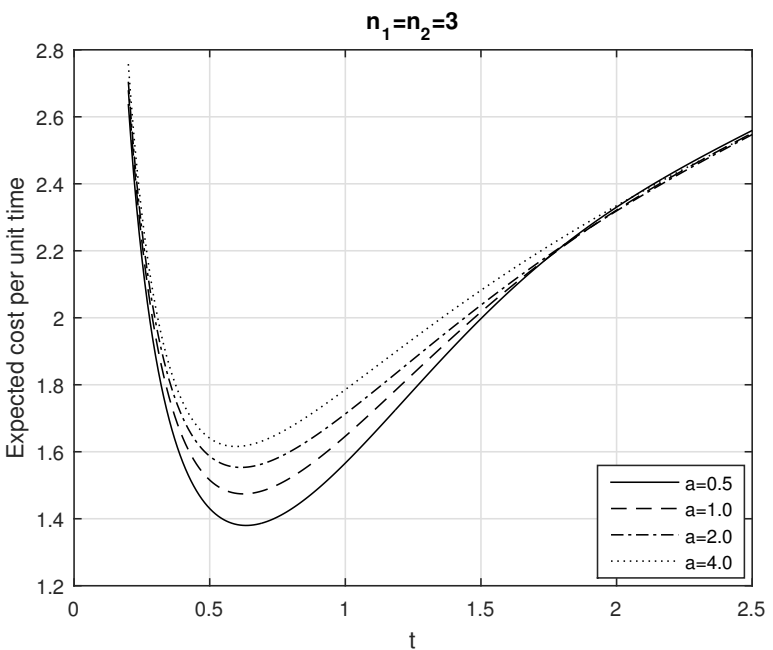

Fig. 6. Expected cost per unit time for ${ }^{\mathrm{t}}$ different repair levels.

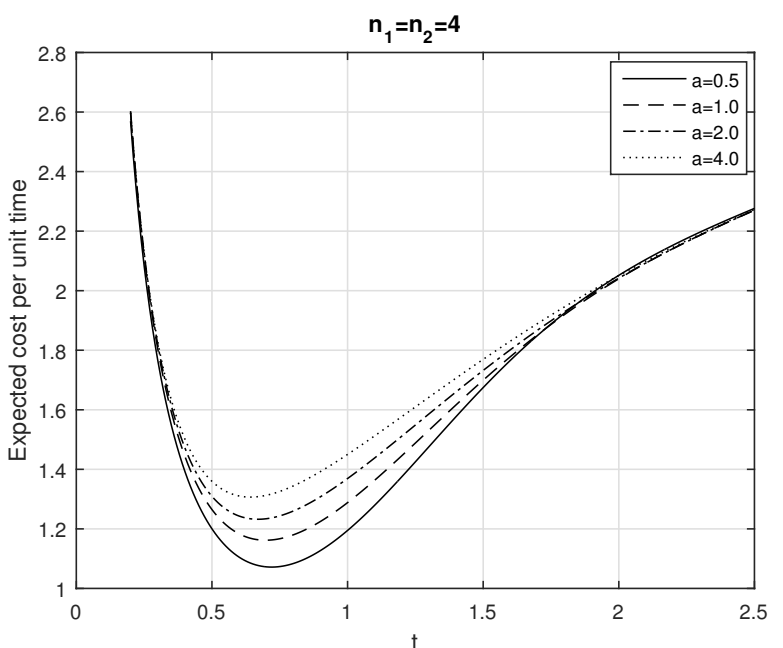

Fig. 7. Expected cost per unit time for ${ }^{\mathrm{t}}$ different repair levels.

TABLE III

OPTIMAL PARAMETERS FOR DIFFERENT PENALTY COSTS $C_{p}$ AND $\left(n_{1}, n_{2}\right)=(4,4)$

\begin{tabular}{llccc}
\hline & \multicolumn{3}{l}{ Optimal maintenance parameters } \\
\cline { 2 - 5 }$C_{p}$ & $\kappa^{*}$ & $\ell^{*}$ & $\tau^{*}$ & $\mathbb{C}_{\tau^{*}}^{\mathbf{0}}$ \\
\hline 0.5 & 5 & 7 & 0.70 & 1.1584 \\
2.5 & 5 & 7 & 0.70 & 1.1599 \\
5.0 & 5 & 7 & 0.70 & 1.1619 \\
7.5 & 5 & 7 & 0.69 & 1.1638 \\
10.0 & 5 & 7 & 0.69 & 1.1656 \\
\hline
\end{tabular}

TABLE IV

OPTIMAL PARAMETERS FOR DIFFERENT REPAIR MODELS AND $\left(n_{1}, n_{2}\right)=(4,4)$.

\begin{tabular}{lcccc}
\hline & \multicolumn{4}{l}{ Optimal maintenance parameters } \\
\cline { 2 - 5 } Repair model & $\kappa^{*}$ & $\ell^{*}$ & $\tau^{*}$ & $\mathbb{C}_{\tau^{*}}^{\mathbf{0}}$ \\
\hline General & 5 & 7 & 0.70 & 1.1619 \\
Variant 1 & - & 7 & 0.83 & 1.6871 \\
Variant 2 & 6 & - & 0.80 & 0.9447 \\
Variant 3 & - & 7 & 1.15 & 1.7900 \\
Variant 4 & - & - & 0.74 & 1.8233 \\
Variant 5 & - & - & 1.21 & 1.5354 \\
Variant 6 & - & - & $\infty$ & 5 \\
\hline
\end{tabular}

TABLE $\mathrm{V}$

OPTIMAL PARAMETERS FOR DIFFERENT REPAIR MODELS AND $\left(n_{1}, n_{2}\right)=(4,4)$.

\begin{tabular}{llccc}
\hline & \multicolumn{3}{l}{ Optimal maintenance parameters } \\
\cline { 2 - 5 } Repair model & $\kappa^{*}$ & $\ell^{*}$ & $\tau^{*}$ & $\mathbb{C}_{\tau^{*}}^{\mathbf{0}}\left(\kappa^{*}, \ell^{*}\right)$ \\
\hline Model I $^{\mathrm{a}}$ & 5 & 7 & 0.76 & 0.7309 \\
Model II $^{\mathrm{b}}$ & 5 & 7 & 0.73 & 0.9641 \\
Model III $^{\mathrm{c}}$ & 5 & 7 & 0.89 & 0.6214 \\
\hline
\end{tabular}

${ }^{\mathrm{a}}$ General repair model given $(a, b)=(0.5,2)$.

${ }^{\mathrm{b}}$ General repair model given $a=b=1$.

${ }^{\mathrm{c}}$ General repair model given $\left(\alpha_{1}, \beta_{1}\right)=\left(\alpha_{2}, \beta_{2}\right)=(1.5,2)$ and $(a, b)=$ $(0.5,2)$. 
variant 2 adapts itself by moving up the optimal partial repair threshold $\left(\kappa^{*}: 5 \mapsto 6\right)$, which results in reducing the frequency of partial repairs and hence reducing costs.

In addition to the six variants of repair models, our proposed model subsumes other repair models, recovered by an appropriate choice of degradation and/or maintenance parameters (see Table V). Model I and Model II, respectively, are referred to the general repair model under the modified repair parameters $(a, b)=(1,0.5)$ and $(a, b)=(1,1)$. The latter assumption turns the kernel transition density into (9). Model III is similar to Model I, but it differs through using only one type of failure model. This allows the maintenance analysis of homogeneous population. As demonstrated, the optimal maintenance thresholds $\left(\kappa^{*}, \ell^{*}\right)$ remains constant in all three cases. The two first rows give an insight into the effect of the partial repair level characterized by the repair parameter $b$. The results reveal that the higher level of repair results in a reduction in both the inspection frequency and the optimal expected cost. A comparative study between the first and the last row reveals that decreasing the failure proneness of components in the first category to the same level of the second category's $\left(\beta_{2}: \sqrt{2} \mapsto 2\right)$ makes inspections less frequent. This results in a reduction in the resulting expected cost.

\section{CONCLUSIONS}

This paper explored an approach to assessing the probability of failure of repairable parallel systems composed of components from heterogeneous populations. The proposed modelling approach differs from others in the sense that it derives a response SDMRL function and associated unprecedented results. This method allows the joint determination of optimal inspection intervals and preventive maintenance policies. The decision process is driven by the excursion of a bivariate state process falling into exclusive subsets determined by maintenance thresholds $(\kappa, \ell)$.

For given parameters, the results provides an insight into the optimal decision process and the behaviour of optimal solutions as the model's parameters change. The model favourably adapts itself to the partial repair level by moving the optimal parameters. It has been shown that the amount of maintenance undertaken on the system decreases when both the redundancy and the partial repair level increase. It is important to notice that the optimal solutions remains almost unchanged by the increase in penalty cost. Also, as the redundancy level remains fixed, changes in the model's parameters makes no changes in optimal replacement threshold $\ell^{*}$ implying a relatively constant frequency of planned replacements.

This paper outlined an approach which can be developed in several directions. Possible future work includes the study of more complex systems characterized by multiple types of subsystems and the extension of the inspection modelling technique to the non-periodic inspection policy implemented through using the response function.

\section{APPENDIX}

\section{A. Proof of Lemma 2}

According to the definition of expectation we have

$$
\begin{aligned}
m(t ; \mathbf{i}) & =\mathbb{E}\left(T_{n: n}-t \mid \mathbf{X}(t)=\mathbf{i}\right)=\int_{0}^{\infty} \mathbb{P}\left(T_{n: n}-t>\omega \mid \mathbf{X}(t)=\mathbf{i}\right) d \omega \\
& =\int_{t}^{\infty} \mathbb{P}\left(T_{n: n}>\omega \mid \mathbf{X}(t)=\mathbf{i}\right) d \omega .
\end{aligned}
$$

Let $i_{0}=\sum_{u=1}^{k} i_{u}$ and $A(v)$ for $v=i_{0}, i_{0}+1, \cdots, n-1$ be the subset of the state space $\Omega$ :

$$
A(v)=\left\{\mathbf{j}: \sum_{u=1}^{k} j_{u}=v: \quad i_{u} \leq j_{u} \leq n_{u} ; u=1,2, \cdots, k\right\} .
$$

Since $\left(T_{n: n}>\omega\right) \equiv(\mathbf{X}(\omega) \in A(v))$ the expectation term (17) becomes

$$
\begin{aligned}
m(t ; \mathbf{i}) & =\int_{t}^{\infty} \mathbb{P}(\mathbf{X}(\omega) \in A(v) \mid \mathbf{X}(t)=\mathbf{i}) d \omega \\
& =\int_{t}^{\infty} \sum_{v=i_{0}} \sum_{A(v)} \mathbb{P}(\mathbf{X}(\omega)=\mathbf{j} \mid \mathbf{X}(t)=\mathbf{i}) d \omega \\
& =\sum_{v=i_{0}}^{n-1} \sum_{A(v)} \int_{t}^{\infty} \prod_{u=1}^{k} \mathscr{B}\left(j_{u}-i_{u} ; n_{u}-i_{u}, 1-\frac{\bar{F}_{u}(\omega)}{\bar{F}_{u}(t)}\right) d \omega .
\end{aligned}
$$

The last line of the proof results from Proposition 1.

\section{B. Proof of Proposition 1}

To facilitate the presentation let $G_{t}(\omega)=\frac{\bar{F}(\omega)}{\bar{F}(t)}$ with respective derivative $g_{t}(\omega)=\frac{d G_{t}(\omega)}{d t}=\lambda(t) \times G_{t}(\omega)$ where $\lambda(t)$ denotes the common failure rate of components. The derivative of $m(t ; i)$ with respect to $t$ yields that

$$
m^{\prime}(t ; i)=-1+\mathbb{A}_{1}+\mathbb{A}_{2}
$$

where

$\mathbb{A}_{1}=\int_{t}^{\infty} g_{t}(\omega) \sum_{j=i}^{n-1}(n-j)\left(\begin{array}{c}n-i \\ j-i\end{array}\right)\left(1-G_{t}(\omega)\right)^{j-i} G_{t}^{n-j-1}(\omega) d \omega$,

and

$\mathbb{A}_{2}=-\int_{t}^{\infty} g_{t}(\omega) \sum_{j=i}^{n-1}(j-i)\left(\begin{array}{c}n-i \\ j-i\end{array}\right)\left(1-G_{t}(\omega)\right)^{j-i-1} G_{t}^{n-j}(\omega) d \omega$.

The terms $\mathbb{A}_{1}$ and $\mathbb{A}_{2}$ can be rewritten as

$$
\begin{aligned}
\mathbb{A}_{1} & =(n-i) \\
& \times \int_{t}^{\infty} g_{t}(\omega) \sum_{j=i}^{n-1}\left(\begin{array}{c}
n-i-1 \\
n-j-1
\end{array}\right)\left(1-G_{t}(\omega)\right)^{j-i} G_{t}^{n-j-1}(\omega) d \omega,
\end{aligned}
$$

and

$$
\begin{aligned}
\mathbb{A}_{2} & =-(n-i) \\
& \times \int_{t}^{\infty} g_{t}(\omega) \sum_{j=i+1}^{n-1}\left(\begin{array}{c}
n-i-1 \\
j-i-1
\end{array}\right)\left(1-G_{t}(\omega)\right)^{j-i-1} G_{t}^{n-j}(\omega) d \omega,
\end{aligned}
$$

By using binomial expansion $\mathbb{A}_{1}$ and $\mathbb{A}_{2}$ become

$\mathbb{A}_{1}=(n-i) \lambda(t) m_{s}(t ; 1)$, 
and

$$
\begin{aligned}
\mathbb{A}_{2} & =-(n-i) \lambda(t) m_{s}(t ; 1) \\
& +(n-i) \lambda(t) \int_{t}^{\infty} \frac{\bar{F}(\omega)}{\bar{F}(t)}\left(1-\frac{\bar{F}(\omega)}{\bar{F}(t)}\right)^{n-i-1} d \omega \\
& =-(n-i) \lambda(t) m_{s}(t ; 1) \\
& +(n-i) \lambda(t) \sum_{x=0}^{n-i-1}\left(\begin{array}{c}
n-i-1 \\
x
\end{array}\right)(-1)^{x} \underbrace{\int_{t}^{\infty}\left(\frac{\bar{F}(\omega)}{\bar{F}(t)}\right)^{x+1} d \omega}_{m_{s}(t ; x+1)} .
\end{aligned}
$$

By plugging $\mathbb{A}_{1}$ and $\mathbb{A}_{2}$ into (17), we get

$m^{\prime}(t ; i)=-1+(n-i) \lambda(t) \sum_{x=0}^{n-i-1}\left(\begin{array}{c}n-i-1 \\ x\end{array}\right)(-1)^{x} m_{s}(t ; x+1)$,

where $m_{s}(t ; x+1)$ denotes the mean residual lifetime of an $(x+1)$-component series system. Since

$$
m_{s}(t ; x+1)=\frac{1+m_{s}^{\prime}(t ; x+1)}{(x+1) \lambda(t)},
$$

(readers are refereed to Ref. [37]) we have

$$
\mathbf{B}(x)=\left(\begin{array}{ccccccc}
1 & 0 & 0 & 0 & 0 & \cdots & 0 \\
2 & -1 & 0 & 0 & 0 & \cdots & 0 \\
3 & -3 & 1 & 0 & 0 & \cdots & 0 \\
4 & -6 & 4 & 1 & 0 & \cdots & 0 \\
\vdots & \vdots & \vdots & \vdots & \vdots & \ddots & \vdots \\
\left(\begin{array}{c}
x \\
i
\end{array}\right) & -\left(\begin{array}{l}
x \\
2
\end{array}\right) & \left(\begin{array}{l}
x \\
3
\end{array}\right) & -\left(\begin{array}{l}
x \\
4
\end{array}\right) & \left(\begin{array}{l}
x \\
5
\end{array}\right) & \cdots & (-1)^{x+1}
\end{array}\right) .
$$

The equivalent system of equations with respect to the SDMRL functions $m(t ; n-i)(i=1,2, \cdots, x)$ can be given by

$$
\begin{aligned}
& m_{s}(t ; 1)=m(t ; n-1) \\
& m_{s}(t ; 2)=2 m(t ; n-1)-m(t ; n-2) \\
& m_{s}(t ; 3)=3 m(t ; n-1)-3 m(t ; n-2)+m(t ; n-3)
\end{aligned}
$$

$m_{s}(t ; x)=\sum_{i=1}^{x}\left(\begin{array}{l}x \\ i\end{array}\right)(-1)^{i+1} m(t ; n-i)$,

or, in a matrix form

$$
\mathbf{m}_{s}(t)=\mathbf{B}(x) \mathbf{m}(t) .
$$

$$
\begin{aligned}
m^{\prime}(t ; i) & =-1+(n-i) \sum_{x=0}^{n-i-1}\left(\begin{array}{c}
n-i-1 \\
x
\end{array}\right)(-1)^{x}\left(\frac{1+m_{s}^{\prime}(t ; x+1)}{x+1}\right) \text { and the proof is complete. } \\
& =-1+\sum_{x=1}^{n-i}\left(\begin{array}{c}
n-i \\
x
\end{array}\right)(-1)^{x+1}\left(1+m_{s}^{\prime}(t ; x)\right), \quad \text { REFERENCES }
\end{aligned}
$$

\section{REFERENCES}

[1] R. Jiang, M.J. Kim and V. Makis. 2019. Availability maximization under partial observations. OR Spectrum; 35(3), 691-710.

Using the fact that $\sum_{x=1}^{n-i}\left(\begin{array}{c}n-i \\ x\end{array}\right)(-1)^{x+1}=1$ we get

$$
m^{\prime}(t ; i)=\sum_{x=1}^{n-i}\left(\begin{array}{c}
n-i \\
x
\end{array}\right)(-1)^{x+1} m_{s}^{\prime}(t ; x) .
$$

Integrating the both side of (18), we obtain

$$
m(t ; i)=\sum_{x=1}^{n-i}\left(\begin{array}{c}
n-i \\
x
\end{array}\right)(-1)^{x+1} m_{s}(t ; x),
$$

and the proof is complete.

\section{Proof of Proposition 2}

Setting $i=n-j$ in (2) we get for $j=1,2, \cdots, x \quad(x=$ $1,2, \cdots, n)$

$$
\begin{aligned}
m(t ; n-1) & =m_{s}(t ; 1) \\
m(t ; n-2) & =2 m_{s}(t ; 1)-m_{s}(t ; 2) \\
m(t ; n-3) & =3 m_{s}(t ; 1)-3 m_{s}(t ; 2)+m_{s}(t ; 3) \\
\vdots & \\
m(t ; n-x) & =\sum_{i=1}^{x}\left(\begin{array}{c}
x \\
i
\end{array}\right)(-1)^{i+1} m_{s}(t ; i) .
\end{aligned}
$$

Or, in the matrix form we have

$$
\mathbf{m}(t)=\mathbf{B}(x) \mathbf{m}_{s}(t)
$$

where $\mathbf{m}(t)=(m(t ; n-i))$ and $\mathbf{m}_{s}(t)=\left(m_{s}(t ; i)\right)$ are vectors of order $x(x=1,2, \cdots, n)$ and $\mathbf{B}(x)=\left(b_{i j}\right) \in Z^{x \times x}$ :
[2] M. Bebbington, C.D. Lai and R. Zitikis. 2008. Reduction in mean residual life in the presence of a constantcompeting risk. Applied Stochastic Models in Business and Industry; 24, 51-63.

[3] J. Navarro and T. Rychlik. 2010. Comparisons andbounds for expected lifetimes of reliability systems. European Journal of Operational Research; 207(1), 309-317.

[4] K.T. Huynh, I.T. Castro, A. Barros and C. Bérenguer. 2012. On the construction of mean residual life for maintenance decision-making. IFAC Proceedings Volumes; 45(20), 654-659.

[5] A.K.S. Jardine, D. Lin and D. Banjevic. 2006. A review on machinery diagnostics and prognostics implementing condition-based maintenance. Mechanical systems and signal processing; 20(7), 1483-1510.

[6] W. Wang and W. Zhang. 2005. A model to predict theresidual life of aircraft engines based upon oil analysisdata. Naval Research Logistics; 52(3), 276-284.

[7] R. Barlow, L. Hunter and F. Proschan. 1963. Optimum cheching procedures. J Soc Ind Appl Math.; 11(4), 10781095.

[8] R. Jiang and A.K.S. Jardine 2004. Two optimization models of the optimum inspection problem. Journal of the Operational Research Society; 56(10), 1176-1183.

[9] A. Chelbi, D. Ait-Kadi and H. Aloui. 2008. Optimal inspection and preventive maintenance policy for systems with self-announcing and non-self-announcing failures. Journal of Quality in Maintenance Engineering; 14(1), 34-45. 
[10] M.D. Berrade. 2012. A two-phase inspection policy with imperfect testing. Applied Mathematical Modelling; 36(1), 108-114.

[11] H.R. Golmakani and H. Moakedi. 2012. Optimal nonperiodic inspection scheme for a multi-component repairable system using $A^{*}$ search algorithm. Computers \& Industrial Engineering; 63(4), 1038-1047.

[12] B. Keleş, S. Tekin and N.O. Bakir. 2017. Maintenance policies for a deteriorating system subject to nonself-announcing failures. IEEE Transactonson Reliabity; 66(1), 219-232.

[13] S.M. Seyedhosseini, H. Moakedi and K. Shahanaghi. 2018. Imperfect inspection optimization for a twocomponent system subject to hidden and two-stage revealed failures over a finite time horizon. Reliability Engineering and System Safety; 174, 141-156.

[14] M.D. Berrade, C. Cavalcante and P. Scarf. 2012. Maintenance scheduling of a protection system subject to imperfect inspection and replacement. European Journal of Operational Research; 218, 716-725.

[15] K. He, L. Maillart and O. Prokopyev. 2015. Scheduling preventive maintenance as a function of an imperfect inspection interval. IEEE Transactions on Reliability; 64(3), 983-997.

[16] V. Babishin and S. Taghipour. 2016. Optimal maintenance policy for multicomponent systems with periodic and opportunistic inspections and preventive replacements. Applied Mathematical Modelling; 40, 1048010505.

[17] V. Babishin and S. Taghipour. 2016. Joint optimal maintenance and inspection for a $k$-out-of- $n$ system. The International Journal of Advanced Manufacturing Technology; 87(5-8), 1739-1749.

[18] R. Ahmadi and S. Wu. 2017. A novel data-driven approach to optimizing replacement policy. Reliability Engineering and System Safety; 167, 506-516.

[19] Q. Qiu, L. Cui and D. Kong. 2019. Availability and maintenance modelling for a two-component system with dependent failures over a finite time horizon. Journal of Risk and Reliability; 233(2), 1-11.

[20] A. Syamsundara, V.N.A. Naikanb and S. Wu. 2020. Alternative scales in reliability models for a repairable system. Reliability Engineering and System Safety; 193, $1-11$.

[21] T. Jianga and Y. Liua. 2020. Selective maintenance strategy for systems executing multiple consecutive missions with uncertainty. Reliability Engineering and System Safety; 193, 1-14.

[22] F. Zhang, J. Shen and Y. Ma. 2020. Optimal maintenance policy considering imperfect repairs and non-constant probabilities of inspection errors. Reliability Engineering and System Safety; 193, 1-12.

[23] K.T. Huynh. 2020. A hybrid condition-based maintenance model for deteriorating systems subject to nonmemoryless imperfect repairs and perfect replacements. IEEE Transactions on Reliability; 69(2), 1-35.

[24] K.T. Huynh. 2020. Modelling past-dependent partial repairs for condition-based maintenance of continuously deteriorating systems. European Journal of Operational Research; 280, 152-163.

[25] W. Kahle. 2019. Imperfect repair in degradation processes: A Kijima type approach. Applied Stochastic Models in Business and Industry; 35(2), 211-220.

[26] S. Mercier and I.T. Castro. 2019. Stochastic comparisons of imperfect maintenance models for a gamma deteriorating system. European Journal of Operational Research; 273, 237-248.

[27] L. Yang, Z.S. Ye, C.G. Lee, S.F. Yang and R. Peng. 2019. A two-phase preventive maintenance policy considering imperfect repair and postponed replacement. European Journal of Operational Research; 274, 966-977.

[28] S. Wu and P. Scarf. 2017. Two new stochastic models of the failure process of a series system. European Journal of Operational Research; 275(3), 763-772.

[29] H. Lee, J. Cha and M. Finkelstein. 2016. On informationbased warranty policy for repairable products from heterogeneous population. European Journal of Operational Research; 253, 204-215.

[30] V. Makis and A.K.S. Jardine. 1992. Optimal replacement policy for a general model with imperfect repair. Journal of the Operational Research Society; 43(2), 111-120.

[31] P.D. Van and C. Bérenguer. 2012. Condition-based maintenance with imperfect preventive repairs for a deteriorating production system. Quality and Reliability Engineering International; 28(6), 624-633.

[32] J.H. Cha, S. Lee and J. Mi. 2004. Comparison of steady system availability with imperfect repair. Applied Stochastic Models in Business and Industry; 20(1), $27-$ 36.

[33] R. Ahmadi. 2020. A new approach to maintenance optimisation of repairable parallel systems subject to hidden failures. Journal of Operational Research Society; 71(9), 1448-1465.

[34] X.Y. Li, Y. Liu, C.J. Chen and T. Jiang. 2015. A copulabased reliability modelling for nonrepairable multi-state $k$-out-of- $n$ systems with dependent components. Journal of Risk and Reliability; 230(2), 1-14.

[35] Q. Qiu, L. Cui and J. Shen. 2018. Availability and maintenance modelling for systems subject to dependent hard and soft failures. Applied Stochastic Models in Business and Industry; 34(4), 513-527.

[36] S. Mercier and H. Pham. 2014. A condition-based imperfect replacement policy for a periodically inspected system with two dependent wear indicators. Applied Stochastic Models in Business and Industry; 30(6), 766782.

[37] M. Rausand and A. Høyland. 2004. System reliability theory: Models, Statistical Methods, and Applications, 2nd Edition (Wiley Series in Probability and Statistics). 